\title{
The effect of perioperative probiotics treatment for colorectal cancer: short-term outcomes of a randomized controlled trial
}

\author{
Yongzhi Yang ${ }^{1, *}$, Yang Xia ${ }^{2,}{ }^{*}$, Hongqi Chen ${ }^{2}$, Leiming Hong ${ }^{1}$, Junlan Feng ${ }^{2}$, Jun \\ Yang ${ }^{2}$, Zhe Yang ${ }^{1}$, Chenzhang Shi ${ }^{1}$, Wen $\mathrm{Wu}^{1}$, Renyuan $\mathrm{Gao}^{1}$, Qing $\mathrm{Wei}^{3}$, Huanlong \\ Qin ${ }^{1}$ and Yanlei $\mathbf{M a}^{1}$ \\ ${ }^{1}$ Department of GI Surgery, Shanghai Tenth People's Hospital Affiliated to Tongji University, Shanghai, China \\ 2 Department of Surgery, Shanghai Jiao Tong University Affiliated Sixth People's Hospital, Shanghai, China \\ ${ }^{3}$ Department of Pathology, Shanghai Tenth People's Hospital Affiliated to Tongji University, Shanghai, China \\ * These authors have contributed equally to this work \\ Correspondence to: Huanlong Qin, email: hl-qin@hotmail.com \\ Yanlei Ma, email: yanleima@live.cn \\ Qing Wei, email: weiqing1971@aliyun.com
}

Keywords: colorectal cancer, perioperative probiotics treatment, post-operative outcome, randomised clinical trial Received: August 03, $2015 \quad$ Accepted: January 13, 2016

Published: January 27, 2016

\section{ABSTRACT}

This study was designed to mainly evaluate the anti-infective effects of perioperative probiotic treatment in patients receiving confined colorectal cancer (CRC) respective surgery. From November 2011 to September 2012, a total of 60 patients diagnosed with CRC were randomly assigned to receive probiotic $(n=30)$ or placebo $(n=30)$ treatment. The operative and post-operative clinical results including intestinal cleanliness, days to first - flatus, defecation, fluid diet, solid diet, duration of pyrexia, average heart rate, length of intraperitoneal drainage, length of antibiotic therapy, blood index changes, rate of infectious and non-infectious complications, postoperative hospital stay, and mortality were investigated. The patient demographics were not significantly different $(p>0.05)$ between the probiotic treated and the placebo groups. The days to first flatus (3.63 versus 3.27 , $p=0.0274)$ and the days to first defecation $(4.53$ versus $3.87, p=0.0268$ ) were significantly improved in the probiotic treated patients. The incidence of diarrhea was significantly lower $(p=0.0352)$ in probiotics group $(26.67 \%, 8 / 30)$ compared to the placebo group $(53.33 \%, 16 / 30)$. There were no statistical differences $(p>0.05)$ in other infectious and non-infectious complication rates including wound infection, pneumonia, urinary tract infection, anastomotic leakage, and abdominal distension. In conclusion, for those patients undergoing confined CRC resection, perioperative probiotic administration significantly influenced the recovery of bowel function, and such improvement may be of important clinical significance in reducing the short-term infectious complications such as bacteremia.

\section{INTRODUCTION}

The amount of gut microbes, which may be nearly 10 times as many as host cells, fluctuates frequently and severely under the circumstances of many gut diseases, such as obesity, irritable bowel syndrome (IBS), inflammatory bowel disease (IBD), and even colorectal cancer (CRC) [1,2]. Dysbiosis of gut bacteria generally occurs in cancer tissues which are directly exposed to microbes, such as colon and rectum [3, 4]. Recent studies confirmed the tight relationship between the microbiota imbalance and cancer progression [5-7]. For example, many opportunistic bacteria species, such as Helicobacter hepaticus, Streptococcus bovis, enterotoxigenic Eschericha coli (ETEC), enterotoxigenic Bacteroides fragilis (ETBF), and Fusobacterium nucleatum, are all confirmed to not only take responsibility for CRC carcinogenesis, but also affect clinical prognosis $[1,8]$.

Postoperative infection is a poor indicator for surgical treatment of cancer [9]. It occurs by a numbers of 
internal and external causes. Intestinal dysbiosis-induced bacterial translocation is the major driver of postoperative infection [9, 10]. Inappropriate use of antibiotics, chemotherapy, or even mechanical bowel preparation for patients undergoing confined colorectal cancer (CRC) resection operation, could also lead to microecological imbalance, subsequently exacerbating the risk for various infections [11]. Hence, probiotics biotherapy emerge as required.

Probiotics are beneficial bacteria that help sustain the homeostasis of gut microenvironment [12]. The ability of both the anti-infection and anti-carcinogen effect of probiotics are mainly based on the following: (a) mutagen binding, competitive inhibition and degradation; (b) host's innate and adaptive immunity enhancement; (c) beneficial gut microbes stimulation and metabolic activity improvement [13]. Therefore, oral administration of probiotics may be beneficial to the patients who are candidates for colorectal surgery $[13,14]$, however, the postoperative clinical benefit in maintaining balance of gut microbiota remains largely unexplored and unknown [15-19]. Our study was designed to primarily evaluate the anti-infective effects of perioperative probiotics treatment in patients receiving confined $\mathrm{CRC}$ resection operation.

\section{MATERIALS AND METHODS}

\section{Ethics approval}

Our study protocol was approved by the ethics committee of the Shanghai sixth People's Hospital affiliated to Shanghai Jiao Tong University. All participants were aware of the aim of this study and signed written informed consent prior to enrollment for random assignment. This trail was registered in www.chictr.org (Registrations number: ChiCTR-TRC-13003332) before participants recruitment started.

\section{Inclusion and exclusion criteria}

Table 1 summarizes various patient inclusion and exclusion criteria for our study.

\section{Participants}

From November 2011 to September 2012, a total of 92 patients diagnosed with sporadic CRC at Shanghai Jiao Tong University Affiliated Sixth People's Hospital were recruited. After criteria eligibility determination, 13 patients were excluded by reason of either refusal to participate $(n=5)$ or matching the inclusion and exclusion criteria $(n=8)$. Of the 79 participants who were randomly assigned to control $(n=37)$ or probiotics group $(n=42)$, 7 and 12 patients, respectively, couldn't complete the study due to either unexecuted assigned intervention or discontinued intervention. At the end of the project, there were 60 eligible patients for statistical analysis, with 30 subjects in each group. Flow diagram of the enrollment and randomization process is illustrated in Figure 1.

\section{Study description}

Randomization was performed in 1:1 proportion, according to a list of randomization numbers. Eligible

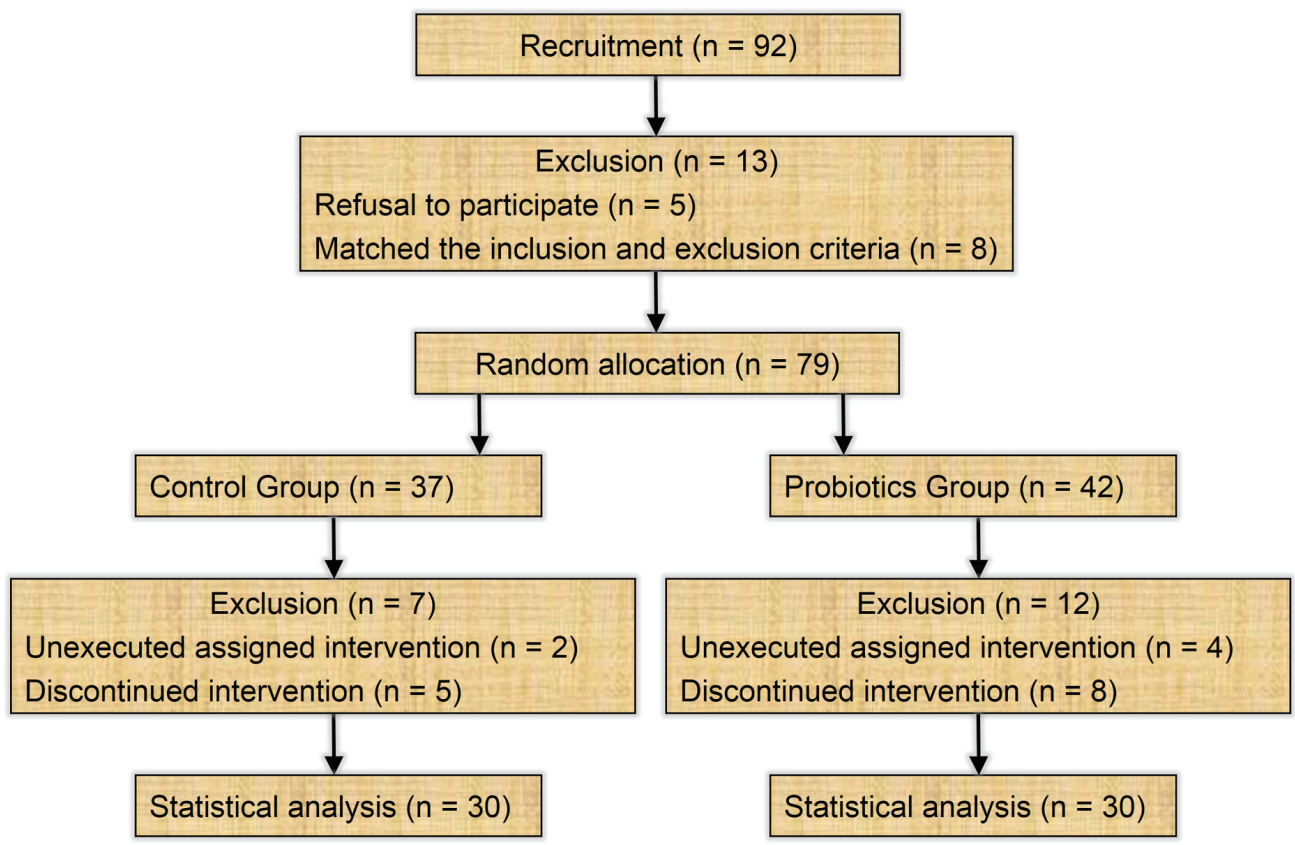

Figure 1: Flow diagram of the enrollment and randomization process. 
Table 1: Inclusion and exclusion criteria for patient enrollment in this clinical trial

\begin{tabular}{|l|l|}
\hline Inclusion criteria & Exclusion criteria \\
\hline Age 25 to 80; & Age more than 80 years old or younger than 25; \\
\hline Received confined colorectal cancer resection operation; & $\begin{array}{l}\text { Co-occurrence of other gastroenterological diseases (e.g. } \\
\text { Inflammatory bowel disease); }\end{array}$ \\
\hline Tolerated curative surgery; & Co-existence of other malignant neoplasms; \\
\hline $\begin{array}{l}\text { Diagnosed with sporadic colorectal cancer by biopsy } \\
\text { examination and family history data collection; }\end{array}$ & $\begin{array}{l}\text { Severe cardiovascular and cerebrovascular diseases that could } \\
\text { not tolerate radical surgery; }\end{array}$ \\
\hline No evidence of cancer metastasis; & Distant metastasis; \\
\hline Joined the trial voluntarily and provided an informed consent. & Recent use of probiotics, prebiotics, or synbiotics; \\
\hline N/A & Recent infection or recent antibiotic use; \\
\hline N/A & Received emergency surgery or laparoscopic surgery; \\
\hline N/A & $\begin{array}{l}\text { Received neoadjuvant chemotherapy, radiotherapy, or } \\
\text { biotherapy; }\end{array}$ \\
\hline N/A & Evidence of Immunodeficiency; \\
\hline N/A & Pregnancy. \\
\hline
\end{tabular}

N/A, not applicable.

patients received either probiotics or placebo treatment ( $2 \mathrm{~g}, \mathrm{po}, \mathrm{tid})$ for 12 consecutive days. Specifically, subjects who were assigned to the probiotics group received combined probiotics (Live combined Bifidobacterium, Lactobacillus and Enterococcus Power, Bifico, Sine Pharmaceuticals, Shanghai, China) containing Bifidobacterium longum $\left(\geq 1.0^{*} 10^{7} \mathrm{cfu} / \mathrm{g}\right)$, Lactobacillus acidophilus $\left(\geq 1.0 * 10^{7} \mathrm{cfu} / \mathrm{g}\right)$, and Enterococcus faecalis $\left(\geq 1.0^{*} 10^{7} \mathrm{cfu} / \mathrm{g}\right) 5$ days before and 7 days after CRC resection operation. This combined probiotics contains three acknowledged medicinal bacterial strain which function as maintaining healthy intestinal flora. When the oral administration was not feasible during the first day after surgery, the probiotics were administered via gastric gavage. Subjects who were assigned to the placebo group received placebo powder containing maltodextrin and sucrose, without any viable probiotics.

Both the probiotics and placebo drugs were packed in the same packaging and were appropriately stored at a controlled condition of $2^{\circ} \mathrm{C}$ to $8^{\circ} \mathrm{C}$. All researchers and subjects were blinded to randomization and treatments during the entire interventional period. Conventional rehydration therapy without additional nutritional supplementations was given to all subjects during the study. For preoperative bowel preparation, all patients received a low-residue diet one day before operation and given 3 litres of polyethylene glycol electrolyte solution the night before surgery. For antibiotic prophylaxis, all patients received one dose of Cefoxitin 30 minutes prior to surgery. After operation, the antibiotic prophylaxis continued when the intraperitoneal drainage had not been removed or whenever the patients developed a fever of over $38.5^{\circ} \mathrm{C}$. All subjects received open colorectal surgery by the same surgeon.

\section{Baseline variables and intraoperative indexes}

The preoperative and intraoperative data of each subject were collected as follows: the gender, age, body mass index (BMI), tumor location, TNM stage, tumor differentiation, preoperative blood indices, length of operation, perioperative bleeding, and intraoperative gut cleanliness. The gut cleanliness scale was assessed according to the recommendation of the previous study [20]. The clinical demographics of eligible Participants are listed in Table 2.

\section{Outcome variables}

The pre-operative and post-operative clinical results, including intra-operative intestinal cleanliness, days to first - flatus, defecation, fluid diet, solid diet, duration of pyrexia, average heart rate, length of intraperitoneal drainage, length of antibiotic therapy, blood index changes, rate of non-infectious complications, infectious complications, postoperative hospital stay, and mortality, were compared. The primary endpoint was the influence of the diarrhea. The influence of the bowel function and the incidence of the other complications were set as secondary endpoints. Clinical definition of main complications is listed in Table 3.

\section{Statistical analysis}

Statistical analysis were conducted using IBM SPSS Statistics 20.0 software (version 20.0, IBM, Inc., Chicago, IL, USA) and GraphPad Prism 6 software (version 6.01, GraphPad software, lnc., San Diego, CA, USA). We used the mean \pm standard deviation (SD) to express the quantitative data. Unpaired Student's t-test was used to 
Table 2: The clinico-pathological characteristics of enrolled participants

\begin{tabular}{|c|c|c|c|}
\hline Characteristic & $\begin{array}{l}\text { Placebo group } \\
(n=30)\end{array}$ & \begin{tabular}{|l|} 
Probiotics group \\
$(\boldsymbol{n}=\mathbf{3 0})$
\end{tabular} & $P$ value \\
\hline $\begin{array}{l}\text { Gender } \\
\text { Female } \\
\text { Male }\end{array}$ & $\begin{array}{l}18 \\
12\end{array}$ & $\begin{array}{l}15 \\
15\end{array}$ & 0.604 \\
\hline Mean Age (years) & $62.17 \pm 11.06$ & $63.90 \pm 12.25$ & 0.567 \\
\hline Body mass index $\left(\mathrm{kg} / \mathrm{m}^{2}\right)$ & $22.07 \pm 1.70$ & $22.13 \pm 1.77$ & 0.895 \\
\hline \multicolumn{4}{|l|}{ Tumor location (n) } \\
\hline Left hemicolon & 14 & 13 & \multirow{3}{*}{0.855} \\
\hline Right hemicolon & 7 & 6 & \\
\hline Rectum & 9 & 11 & \\
\hline \multicolumn{4}{|l|}{ TNM stage (n) } \\
\hline 0 - II & 21 & 23 & \multirow{2}{*}{0.771} \\
\hline III & 9 & 7 & \\
\hline \multicolumn{4}{|l|}{ Tumor differentiation (n) } \\
\hline Well-moderate & 27 & 25 & \multirow{2}{*}{0.707} \\
\hline Poor & 3 & 5 & \\
\hline \multicolumn{4}{|l|}{ Preoperative blood index } \\
\hline Leucocyte $\left(* 10^{9} / \mathrm{L}\right)$ & $6.56 \pm 2.54$ & $6.14 \pm 1.47$ & 0.437 \\
\hline Hemoglobin $(\mathrm{g} / \mathrm{L})$ & $116.20 \pm 21.46$ & $121.3 \pm 22.08$ & 0.371 \\
\hline Albumin $(\mathrm{g} / \mathrm{L})$ & $38.5 \pm 6.51$ & $40.43 \pm 5.41$ & 0.216 \\
\hline Creatinine (umol/L) & $67.1 \pm 18.74$ & $72.24 \pm 22.85$ & 0.345 \\
\hline Glucose (mmol/L) & $5.43 \pm 1.03$ & $5.39 \pm 0.67$ & 0.858 \\
\hline Triglyceride $(\mathrm{mmol} / \mathrm{L})$ & $1.56 \pm 0.91$ & $1.27 \pm 0.50$ & 0.353 \\
\hline Total cholesterol $(\mathrm{mmol} / \mathrm{L})$ & $4.36 \pm 0.98$ & $3.95 \pm 1.36$ & 0.530 \\
\hline Length of operation (min) & $128.34 \pm 36.58$ & $128.12 \pm 28.31$ & 0.979 \\
\hline Perioperative bleeding (mL) & $116.60 \pm 29.79$ & $123.40 \pm 30.50$ & 0.388 \\
\hline \multicolumn{4}{|c|}{ Intraoperative gut cleanliness (n) } \\
\hline Excellent & 12 & 19 & \multirow{3}{*}{0.073} \\
\hline Good or fair & 16 & 10 & \\
\hline Fair & 2 & 1 & \\
\hline
\end{tabular}

compare the continuous variables. Categorical variables were analyzed using the Pearson's Chi-square test and Fisher's exact test, as appropriate. Ordinal variables were analyzed using Mann-Whitney U test. A power of $80 \%$ and an alpha error of $5 \%$ were set to approximately calculated the sample size. All tests were double tail and the $p$ values below 0.05 were considered statistically significant.

\section{RESULTS}

A total of 30 participants were included in each group. The general demographics for the two groups of participants are presented in Table 2. There were no significant differences with regard to gender $(p=0.604)$, age $(p=0.567)$, BMI $(p=0.895)$, tumor location $(p=$ $0.855)$, TNM stage ( $p=0.771)$, and tumor differentiation $(p=0.707)$ between the probiotics and the placebo groups. Moreover, no significant differences were found between the two groups in term of the blood indices 5 days before surgery, including leucocyte count $(p=0.437)$, and levels of hemoglobin $(p=0.371)$, albumin $(p=0.216)$, creatinine $(p=0.345)$, glucose $(p=0.858)$, triglyceride $(p=0.353)$, and total cholesterol $(p=0.530)$, suggesting that the baseline of the two groups was quite homogeneous. 
Table 3: Clinical definition of complications

\begin{tabular}{|l|l|}
\hline Complications & Clinical definition \\
\hline Pyrexia & Oral temperature higher than $38.5^{\circ} \mathrm{C}$. \\
\hline Infection & 7 day blood-cultures positive. \\
\hline Bacteremia & $\begin{array}{l}\text { Surgical site suppuration and the bacterial cultures of purulent exudate } \\
\text { positive. }\end{array}$ \\
\hline Wound infection & $\begin{array}{l}\text { A typical pulmonary infiltrate can be seen on a chest X-ray and/or the } \\
\text { swab culture is positive. }\end{array}$ \\
\hline Pneumonia & $\begin{array}{l}\text { There are obvious symptoms including frequent micturition, urgency to } \\
\text { urinate, and urodynia, accompanied by bacteriuria (100,000 cfu/mL). }\end{array}$ \\
\hline Urinary tract infection & $\begin{array}{l}\text { Presence of any of the following clinical signs: fecal discharge from the } \\
\text { wound or drain, circumscribed abcess near the site of anastomosis, or } \\
\text { fecal peritonitis confirmed by CT. }\end{array}$ \\
\hline Anastomotic leakage & \\
\hline Bowel dysfunction & $\begin{array}{l}\text { There is a symptom of having loose or liquid feces more than } 3 \text { times } \\
\text { a day. }\end{array}$ \\
\hline Diarrhea & There is a sense of abdominal pressure or fullness. \\
\hline Abdominal distension & The occurrence of death during hospitalization \\
\hline Mortality &
\end{tabular}

Abbreviation: cfu, colony-forming unit; CT, computed tomography.

With regard to the intraoperative data, the length of operation $(p=0.979)$ and perioperative bleeding ( $p=$ 0.388 ) were similar between the two groups. It's worth mentioning that the intraoperative intestinal cleanliness had slight difference, although these results did not reach statistical significance $(p=0.073)$. This finding implied that perioperative probiotics treatment could likely be of tremendous clinical benefit as a supplement during bowel preparation in patients prepared for confined CRC surgery.

The Table 4 summarizes the information on postoperative short-term outcomes between the probiotics group and the placebo group. No significant differences $(p>0.05)$ were found between the placebo $v s$. probiotics groups in terms of days to first fluid diet (3.93 $\pm 0.78 \mathrm{vs}$. $3.73 \pm 0.83$ days; $p=0.341)$, days to first solid diet $(5.00$ \pm 0.83 vs. $4.87 \pm 0.86$ days; $p=0.544)$, duration of pyrexia $(4.80 \pm 2.34$ vs. $4.77 \pm 1.79$ days; $p=0.951)$, average heart rate in a week after surgery $(78.98 \pm 3.78 v s .80 .63 \pm 4.13$ bpm; $p=0.111)$, length of intraperitoneal drainage $(6.67 \pm$ $1.09 v s .6 .50 \pm 0.97$ days; $p=0.535)$, length of antibiotic therapy $(7.33 \pm 3.86 v s .6 .60 \pm 2.81$ days; $p=0.404)$, and postoperative hospital stay $(15.00 \pm 4.31 \mathrm{vs} .15 .86 \pm 4.92$ days; $p=0.487)$. However, the days to first flatus (3.63 \pm 0.67 days in the placebo group versus $3.27 \pm 0.58$ days in the probiotics group, $p=0.0274$ ) and the days to first defecation ( $4.53 \pm 1.11$ days in the placebo group versus $3.87 \pm 1.17$ days in the probiotics group, $p=0.0268$ ) were significantly improved in the probiotics group. These findings imply that the probiotics treatment resulted in a faster recovery of bowel function for patients with CRC operation.

Blood indices related to blood routine and hepatorenal function was collected 5 days before and 7 days after surgery. All blood index changes were negative in the placebo group compared with the probiotics group, such as white blood cell $\left(2.11 \pm 2.26 * 10^{9} / \mathrm{L} v s .1 .64 \pm\right.$ $\left.1.78 * 10^{9} / \mathrm{L}, p=0.374\right)$, hemoglobin $(14.73 \pm 11.44 \mathrm{~g} / \mathrm{L} v s$. $14.50 \pm 10.58 \mathrm{~g} / \mathrm{L}, p=0.935)$, albumin $(7.93 \pm 5.37 \mathrm{~g} / \mathrm{L}$ vs. $6.69 \pm 4.40 \mathrm{~g} / \mathrm{L}, p=0.336)$, creatinine $(14.13 \pm 12.34$ umol/L vs. $16.07 \pm 11.36 \mathrm{umol} / \mathrm{L}, p=0.534)$, glucose $(1.39 \pm 1.69 \mathrm{mmol} / \mathrm{L} v s .1 .04 \pm 1.36 \mathrm{mmol} / \mathrm{L}, p=0.541)$, triglyceride $(0.35 \pm 0.33 \mathrm{mmol} / \mathrm{L}$ vs. $0.13 \pm 0.04 \mathrm{mmol} / \mathrm{L}$, $p=0.136)$, and total cholesterol $(0.69 \pm 0.63 \mathrm{mmol} / \mathrm{L} v s$. $0.79 \pm 0.46 \mathrm{mmol} / \mathrm{L}, p=0.773)$.

We than compared the non-infectious and the infectious complications in the two groups. The incidence of diarrhea was significantly lower $(p=0.0352)$ in probiotics group $(26.67 \%, 8 / 30)$ compared to the placebo group $(53.33 \%, 16 / 30)$, whereas other non-infectious complications including anastomotic leakage (1 incident in the placebo group vs. 1 incidence in the probiotics group, $p=1.000$ ), and abdominal distension (13 incidents in the placebo group vs. 9 incidents in the probiotics group, $p=$ 0.284 ) were essentially quite comparable.

As for the infectious complications, although the incidence of bacteremia was slightly lower in probiotics group $(30 \%, 9 / 30)$ than in placebo group $(10 \%, 3 / 30)$, the difference didn't reach statistical significance $(p=0.0528)$. There were no statistical differences $(p>0.05)$ in other infectious complications rate. For example, the wound infection rate ( 1 incidence in each group respectively, $p$ $=1.000)$ and the urinary tract infection rate $(2$ incidences in each group respectively, $p=0.605$ ) were identical in the two groups. 5 Pneumonia complications occurred in the placebo group, whereas 3 incidents occurred in the probiotics group $(p=0.704)$. No side-effect of drug or mortality occurred in either group. 
Table 4: Postoperative short-term outcomes

\begin{tabular}{|c|c|c|c|}
\hline Postoperative outcomes & $\begin{array}{l}\text { Placebo group } \\
(n=30)\end{array}$ & $\begin{array}{l}\text { Probiotics group }(n= \\
\text { 30) }\end{array}$ & $P$ value \\
\hline Days to first flatus (d) & $3.63 \pm 0.67$ & $3.27 \pm 0.58$ & 0.0274 \\
\hline Days to first defecation (d) & $4.53 \pm 1.11$ & $3.87 \pm 1.17$ & 0.0268 \\
\hline Days to first fluid diet (d) & $3.93 \pm 0.78$ & $3.73 \pm 0.83$ & 0.341 \\
\hline Days to first solid diet (d) & $5.00 \pm 0.83$ & $4.87 \pm 0.86$ & 0.544 \\
\hline Duration of pyrexia $\left(>38.5^{\circ} \mathrm{C}, \mathrm{d}\right)$ & $4.80 \pm 2.34$ & $4.77 \pm 1.79$ & 0.951 \\
\hline Average heart rate (in a week after surgery, bpm) & $78.98 \pm 3.78$ & $80.63 \pm 4.13$ & 0.111 \\
\hline Length of intraperitoneal drainage (d) & $6.67 \pm 1.09$ & $6.50 \pm 0.97$ & 0.535 \\
\hline Length of antibiotic therapy (d) & $7.33 \pm 3.86$ & $6.60 \pm 2.81$ & 0.404 \\
\hline Postoperative hospital stay (d) & $15.00 \pm 4.31$ & $15.86 \pm 4.92$ & 0.487 \\
\hline \multicolumn{4}{|l|}{ Blood index change } \\
\hline Leucocyte $\left(* 10^{9} / \mathrm{L}\right)$ & $2.11 \pm 2.26$ & $1.64 \pm 1.78$ & 0.374 \\
\hline Hemoglobin (g/L) & $14.73 \pm 11.44$ & $14.50 \pm 10.58$ & 0.935 \\
\hline Albumin $(\mathrm{g} / \mathrm{L})$ & $7.93 \pm 5.37$ & $6.69 \pm 4.40$ & 0.336 \\
\hline Creatinine (umol/L) & $14.13 \pm 12.34$ & $16.07 \pm 11.36$ & 0.534 \\
\hline Glucose (mmol/L) & $1.39 \pm 1.69$ & $1.04 \pm 1.36$ & 0.541 \\
\hline Triglyceride (mmol/L) & $0.35 \pm 0.33$ & $0.13 \pm 0.04$ & 0.136 \\
\hline Total cholesterol (mmol/L) & $0.69 \pm 0.63$ & $0.79 \pm 0.46$ & 0.773 \\
\hline \multicolumn{4}{|l|}{ Incidence of Infectious complications (\%) } \\
\hline Bacteremia (\%) & $30.00(9 / 30)$ & $10 \%(3 / 30)$ & 0.0528 \\
\hline Wound infection (\%) & $3.33(1 / 30)$ & $3.33(1 / 30)$ & 1.000 \\
\hline Pneumonia (\%) & $16.67(5 / 30)$ & $10.00(3 / 30)$ & 0.704 \\
\hline Urinary tract infection (n) & $6.67(2 / 30)$ & $6.67(2 / 30)$ & 0.605 \\
\hline \multicolumn{4}{|l|}{ Incidence of non-infectious complications (\%) } \\
\hline Anastomotic leakage (\%) & $96.67(1 / 30)$ & $93.33(2 / 30)$ & 1.000 \\
\hline Diarrhea $(\%)$ & $53.33(16 / 30)$ & $26.67(8 / 30)$ & 0.0352 \\
\hline Abdominal distension ( $\%)$ & $43.33(13 / 30)$ & $30.00(9 / 30)$ & 0.284 \\
\hline Mortality (n) & $\mathrm{N} / \mathrm{A}$ & $\mathrm{N} / \mathrm{A}$ & $\mathrm{N} / \mathrm{A}$ \\
\hline
\end{tabular}

N/A, not applicable. 


\section{DISCUSSION}

The successfully use of probiotics have been reported to help administrate several functional gut disorders [14]. Thus, probiotics treatment for cancerrelated gut complications would be of great interest. It is widely accepted that both surgical and nonsurgical treatments can frequently lead to gastrointestinal symptoms for patients with cancer [21]. Postoperative gastrointestinal symptoms such as diarrhea increase the risk of malnutrition, infectious complications, and longer hospitalization in patients with CRC surgery [20]. In our current study, we observed a significantly lower incidence of diarrhea $(p=0.0352)$ in probiotics group than in placebo group. In addition, in patients with the treatment of probiotics, the days to first flatus $(p=0.0274)$ or defecation $(p=0.0268)$ was earlier, implying faster recovery of bowel function. Such improvements may attribute to the effects of combined probiotics on the host physiology, including metabolism, intestinal function, bone homeostasis, and even emotion and behavior [1]. One of our recent study have already confirmed the effect of this combined probiotics on anti-inflammatory, regulation of immunity, and maintenance of gut barrier integrity in a interleukin-10 deficient mice model and Caco-2 cell line [22]. Our present study also confirmed the findings reported by a previous systematic reviews, which supported the potential benefit of probiotics in reducing diarrhea and sepsis rate in patients with cancer $[15,17,23]$. The relatively high incidence of diarrhea in our subjects may attribute to the open surgical proceure and high proportion of left semi-colorectal carcinoma.

Better bowel preparation may improve the disease outcome [24]. We found that after perioperative probiotics treatment, the intraoperative intestinal cleanliness had slight improvement. Whereas, one review study reported by peitsidou et al [19] did not advocate the combined use of beneficial microecologics and mechanical bowel preparation in patients with CRC operation. Thus, further studies, including larger patient cohort and longer probiotics use, are required to further confirm our results.

Several studies have assessed and supported the positive benefit for maintaining the intestinal microbiota balance by perioperative probiotics treatment in patients undergoing biliary Cancer Surgery, pancreatic duodenectomy, liver transplantation, and coloproctectomy [25-32]. Our previous study also supported the gut barrier protective function of perioperative probiotics treatment [33]. In our current study, the incidence of bacteremia was slightly lower in probiotics group than in placebo group $(p=0.0528)$. Our study suggests that, with shortterm follow-up, perioperative probiotics administration significantly influenced the recovery of bowel function and such improvement may be of tremendous clinical value in reducing the infectious complications such as bacteremia or even gut-origin sepsis.
One limitation of this study was the relative short period of the use of probiotics. The other limitation was the absence of continuous administration of probiotics after hospital discharge in these subjects. It would be appropriate for follow-up to assess the benefits of long-term probiotics use for these subjects receiving chemotherapy.

\section{CONCLUSIONS}

In conclusion, our study provides first-hand clinical evidence that perioperative probiotic administration may help those patients undergoing confined CRC resection surgery in obtaining short-term clinical benefit considering faster recovery of bowel function, lower incidences of diarrhea, and slightly lower rate of bacteremia.

\section{FUNDING}

This work was financially sponsored by grants from the National Natural Science Foundation of China (No.81230057; No.81472262; No.81372615), the National High Technology Research and Development Program (863 Program; Grant No. 2014AA020803), the National Science and Technology Major Projects (2013ZX09103003-16), Shanghai Health System Outstanding Young Talent Training Plan (No. XYQ2013118).

\section{CONFLICTS OF INTERESTS}

The authors declare no relevant competing financial interests to disclose.

\section{REFERENCES}

1. Sommer F and Backhed F. The gut microbiota-masters of host development and physiology. Nature reviews Microbiology. 2013; 11:227-238.

2. Ursell LK, Metcalf JL, Parfrey LW and Knight R. Defining the human microbiome. Nutrition reviews. 2012; 70 Suppl 1:S38-44.

3. Tjalsma H, Boleij A, Marchesi JR and Dutilh BE. A bacterial driver-passenger model for colorectal cancer: beyond the usual suspects. Nature reviews Microbiology. 2012; 10:575-582.

4. Worthley DL, Le Leu RK, Whitehall VL, Conlon M, Christophersen C, Belobrajdic D, Mallitt KA, Hu Y, Irahara $\mathrm{N}$, Ogino S, Leggett BA and Young GP. A human, double-blind, placebo-controlled, crossover trial of prebiotic, probiotic, and synbiotic supplementation: effects on luminal, inflammatory, epigenetic, and epithelial biomarkers of colorectal cancer. The American journal of clinical nutrition. 2009; 90:578-586.

5. Hold GL and Garrett WS. Gut microbiota: Microbiota 
organisation-a key to understanding CRC development. Nature reviews Gastroenterology \& hepatology. 2015.

6. Leung A, Tsoi H and Yu J. Fusobacterium and Escherichia: models of colorectal cancer driven by microbiota and the utility of microbiota in colorectal cancer screening. Expert review of gastroenterology \& hepatology. 2015:1-7.

7. Lokody I. Tumour microenvironment: bacterial balance affects cancer treatment. Nature reviews Cancer. 2014; $14: 10$.

8. Sears CL and Garrett WS. Microbes, Microbiota, and Colon Cancer. Cell host \& microbe. 2014; 15:317-328.

9. Salvans S, Mayol X, Alonso S, Messeguer R, Pascual M, Mojal S, Grande L and Pera M. Postoperative peritoneal infection enhances migration and invasion capacities of tumor cells in vitro: an insight into the association between anastomotic leak and recurrence after surgery for colorectal cancer. Annals of surgery. 2014; 260:939-943; discussion 943-934.

10. Deitch EA. Gut-origin sepsis: evolution of a concept. Surgeon. 2012; 10:350-356.

11. Jalanka J, Salonen A, Salojarvi J, Ritari J, Immonen O, Marciani L, Gowland P, Hoad C, Garsed K, Lam C, Palva A, Spiller RC and de Vos WM. Effects of bowel cleansing on the intestinal microbiota. Gut. 2014.

12. Serban DE. Gastrointestinal cancers: influence of gut microbiota, probiotics and prebiotics. Cancer letters. 2014; 345:258-270.

13. Raman M, Ambalam P, Kondepudi KK, Pithva S, Kothari C, Patel AT, Purama RK, Dave JM and Vyas BR. Potential of probiotics, prebiotics and synbiotics for management of colorectal cancer. Gut microbes. 2013; 4:181-192.

14. Azcarate-Peril MA, Sikes M and Bruno-Barcena JM. The intestinal microbiota, gastrointestinal environment and colorectal cancer: a putative role for probiotics in prevention of colorectal cancer? American journal of physiology Gastrointestinal and liver physiology. 2011; 301:G401-424.

15. Redman MG, Ward EJ and Phillips RS. The efficacy and safety of probiotics in people with cancer: a systematic review. Ann Oncol. 2014; 25:1919-1929.

16. Sanders ME, Guarner F, Guerrant R, Holt PR, Quigley EM, Sartor RB, Sherman PM and Mayer EA. An update on the use and investigation of probiotics in health and disease. Gut. 2013; 62:787-796.

17. He D, Wang HY, Feng JY, Zhang MM, Zhou Y and Wu $\mathrm{XT}$. Use of pro-/synbiotics as prophylaxis in patients undergoing colorectal resection for cancer: a meta-analysis of randomized controlled trials. Clinics and research in hepatology and gastroenterology. 2013; 37:406-415.

18. Okazaki M, Matsukuma S, Suto R, Miyazaki K, Hidaka M, Matsuo M, Noshima S, Zempo N, Asahara T and Nomoto K. Perioperative synbiotic therapy in elderly patients undergoing gastroenterological surgery: a prospective, randomized control trial. Nutrition. 2013; 29:1224-1230.
19. Peitsidou K, Karantanos $\mathrm{T}$ and Theodoropoulos GE. Probiotics, prebiotics, synbiotics: is there enough evidence to support their use in colorectal cancer surgery? Digestive surgery. 2012; 29:426-438.

20. Johnson DA, Barkun AN, Cohen LB, Dominitz JA, Kaltenbach T, Martel M, Robertson DJ, Boland CR, Giardello FM, Lieberman DA, Levin TR, Rex DK and Cancer USM-STFoC. Optimizing adequacy of bowel cleansing for colonoscopy: recommendations from the US multi-society task force on colorectal cancer. Gastroenterology. 2014; 147:903-924.

21. Blencowe NS, Chana P, Whistance RN, Stevens D, Wong NA, Falk SJ and Blazeby JM. Outcome reporting in neoadjuvant surgical trials: a systematic review of the literature and proposals for new standards. Journal of the National Cancer Institute. 2014; 106.

22. Shi CZ, Chen HQ, Liang Y, Xia Y, Yang YZ, Yang J, Zhang JD, Wang SH, Liu J and Qin HL. Combined probiotic bacteria promotes intestinal epithelial barrier function in interleukin-10-gene-deficient mice. World J Gastroenterol. 2014; 20:4636-4647.

23. Pitsouni E, Alexiou V, Saridakis V, Peppas G and Falagas ME. Does the use of probiotics/synbiotics prevent postoperative infections in patients undergoing abdominal surgery? A meta-analysis of randomized controlled trials. European journal of clinical pharmacology. 2009; 65:561570.

24. Kim EK, Sheetz KH, Bonn J, DeRoo S, Lee C, Stein I, Zarinsefat A, Cai S, Campbell DA, Jr. and Englesbe MJ. A statewide colectomy experience: the role of full bowel preparation in preventing surgical site infection. Annals of surgery. 2014; 259:310-314.

25. Sugawara G, Nagino M, Nishio H, Ebata T, Takagi K, Asahara $\mathrm{T}$, Nomoto $\mathrm{K}$ and Nimura $\mathrm{Y}$. Perioperative synbiotic treatment to prevent postoperative infectious complications in biliary cancer surgery: a randomized controlled trial. Annals of surgery. 2006; 244:706-714.

26. Nomura T, Tsuchiya $\mathrm{Y}$, Nashimoto A, Yabusaki H, Takii Y, Nakagawa S, Sato N, Kanbayashi C and Tanaka O. Probiotics reduce infectious complications after pancreaticoduodenectomy. Hepato-gastroenterology. 2007; 54:661-663.

27. Rayes N, Seehofer D, Theruvath T, Schiller RA, Langrehr JM, Jonas S, Bengmark S and Neuhaus P. Supply of pre- and probiotics reduces bacterial infection rates after liver transplantation - a randomized, double-blind trial. American journal of transplantation. 2005; 5:125-130.

28. Sommacal HM, Bersch VP, Vitola SP and Osvaldt AB. Perioperative synbiotics decrease postoperative complications in periampullary neoplasms: a randomized, double-blind clinical trial. Nutrition and cancer. 2015; 67:457-462.

29. Liu Z, Li C, Huang M, Tong C, Zhang X, Wang L, Peng H, Lan P, Zhang P, Huang N, Peng J, Wu X, Luo Y, Qin $\mathrm{H}$, Kang L and Wang J. Positive regulatory effects of 
perioperative probiotic treatment on postoperative liver complications after colorectal liver metastases surgery: a double-center and double-blind randomized clinical trial. BMC gastroenterology. 2015; 15:34.

30. Bellows CF, Mills KT, Kelly $\mathrm{TN}$ and Gagliardi G. Combination of oral non-absorbable and intravenous antibiotics versus intravenous antibiotics alone in the prevention of surgical site infections after colorectal surgery: a meta-analysis of randomized controlled trials. Techniques in coloproctology. 2011; 15:385-395.

31. Liu ZH, Huang MJ, Zhang XW, Wang L, Huang NQ, Peng H, Lan P, Peng JS, Yang Z, Xia Y, Liu WJ, Yang J, Qin HL and Wang JP. The effects of perioperative probiotic treatment on serum zonulin concentration and subsequent postoperative infectious complications after colorectal cancer surgery: a double-center and double-blind randomized clinical trial. The American journal of clinical nutrition. 2013; 97:117-126.

32. Usami M, Miyoshi M, Kanbara Y, Aoyama M, Sakaki H, Shuno K, Hirata K, Takahashi M, Ueno K, Tabata S, Asahara $\mathrm{T}$ and Nomoto K. Effects of perioperative synbiotic treatment on infectious complications, intestinal integrity, and fecal flora and organic acids in hepatic surgery with or without cirrhosis. JPEN Journal of parenteral and enteral nutrition. 2011; 35:317-328.

33. Liu Z, Qin H, Yang Z, Xia Y, Liu W, Yang J, Jiang Y, Zhang H, Yang Z, Wang Y and Zheng Q. Randomised clinical trial: the effects of perioperative probiotic treatment on barrier function and post-operative infectious complications in colorectal cancer surgery - a double-blind study. Alimentary pharmacology \& therapeutics. 2011; 33:50-63. 